\title{
Parametric resonances of floating wind turbine blades under vertical wave excitation
}

\author{
Takashi Ikeda ${ }^{1, *}$, Yuji Harata $^{2}$, Yugo Miyazawa ${ }^{3}$, and Yukio Ishida ${ }^{4}$ \\ ${ }^{1}$ Department of Mechanical Systems Engineering, Hiroshima University, 1-4-1 Kagamiyama, \\ Higashi-Hiroshima, Hiroshima, Japan \\ ${ }^{2}$ Department of Mechanical Engineering, Aichi Institute of Technology, 1247 Yachigusa, Yakusa-cho, \\ Toyota, Aichi, Japan \\ ${ }^{3}$ Kao Corporation, 1-14-10 Kayaba-cho, Nihonbashi, Chuo-ku, Tokyo, Japan \\ ${ }^{4}$ Institute of International Education and Exchange, Nagoya University, Furo-cho, Chikusa-ku, \\ Nagoya, Aichi, Japan
}

\begin{abstract}
The parametric resonances of the blades in floating offshore wind turbines are theoretically and experimentally investigated. In the theoretical analysis, each blade is pinned to a horizontal, rotating shaft and has a spring with rotational stiffness at the end. The blade is subjected to horizontal excitation which represents winds; the rotating shaft to vertical excitation which represents waves. The equation of motion for the blade inclination angle includes parametric excitation terms with three different frequencies, i.e., the rotational speed of the blade, and the sum of and difference between the rotational speed and wave excitation frequency. Numerical simulations are conducted for the corresponding linearized system, and it is found that unstable vibrations appear at several rotational speed ranges. An empirical approach is used to determine the regions where the unstable vibrations appear. Swept-sine tests are conducted to determine the frequency response curves for the nonlinear system and demonstrate that the parametric resonances appear at similar rotational speeds as those of the unstable regions. In experiments, parametric resonances were observed at the rotational speeds and wave excitation frequencies predicted by the theoretical analysis.
\end{abstract}

\section{Introduction}

In order to solve energy problems such as the depletion of the limited supply of fossil fuels and the safety of nuclear power generation, cleaner energy sources are being developed. One such source is wind power generation. Although most wind power is generated from land-based wind turbines, floating offshore wind turbines are attracting attention because they can generate comparatively constant wind power throughout the year. Due to severe meteorological conditions, there are limited demonstration tests that verify the safety of such turbines [1]. There are also few academic studies on the vibrations of floating offshore wind turbines, despite the influence such research could have on their commercialization.

This paper investigates the parametric resonances of floating offshore wind turbine blades. In the theoretical analysis, a model is used in which the blade is subjected to

\footnotetext{
*Corresponding author: tikeda@hiroshima-u.ac.jp
} 
horizontal wind excitation, and a rotating shaft to vertical, harmonic excitation, which represents the motion of waves. In the numerical simulations for the corresponding linearized system, unstable vibrations are observed because the equation of motion for the inclination angle of the blade includes parametric excitation terms. Then, the regions where the unstable vibrations appear are theoretically determined. When the nonlinearity of the blades is considered, the swept-sine test is performed to calculate frequency response curves. These curves are then compared to the theoretical results of the linearized system. Experiments are also conducted to confirm the validity of the theoretical analysis.

\section{Theoretical analysis}

\subsection{Equation of motion}

Figure 1 shows a spar-type floating wind turbine consisting of a spar buoy, tower, nacelle and three blades. The blades are subjected to winds, and the spar buoy is subjected to wave motion. In the orthogonal stationary coordinate system $\mathrm{O}-x y z$, the $z$-axis coincides with the wind direction and a horizontal rotating shaft of the blade in the nacelle.

Figure 2 shows the theoretical model of an arbitrary blade. The effect of gravity on the stability of a rotating cantilever beam in a vertical plane was investigated [2]. However, here, for simplicity, the blade is modeled as a rigid flat plate with length $l$, width $b$, thickness $h$, and mass $m=\rho l b h$ ( $\rho$ : density). The blade is assumed to be pinned to a horizontal, rotating shaft at point $\mathrm{P}$ and has a spring with rotational stiffness $k$ at point $\mathrm{P}$. The inclination angle of the blade is designated as $\theta$, which is measured from the vertical plane O-xy. The blade is subjected to horizontal wind excitation, which has vertical, uniform wind speeds $V_{0}$. The rotating shaft is subjected to vertical, harmonic excitation $y_{P}=b_{y} \sin \Omega t$, hereafter referred to as wave excitation. Lagrange's equation is employed to obtain the equation of motion for the blade as follows:

$$
\frac{m l^{2}}{3} \ddot{\theta}+c \dot{\theta}+k \theta-\frac{m l}{2}\left(g-b_{y} \Omega^{2} \sin \Omega t\right) \sin \omega t \sin \theta+\frac{m l^{2} \omega^{2}}{6} \sin 2 \theta-\frac{\rho_{a} b l^{2} V_{0}^{2}}{2} \cos ^{2} \theta=0,
$$

where $\omega$ is the rotational speed of the blade, $c$ is the damping coefficient, $g$ is the acceleration of gravity, and $\rho_{a}$ is the air density. Equation (1) includes parametric excitation terms with three different frequencies, i.e., $\omega, \omega+\Omega$, and $\omega-\Omega$. The dimensionless quantities

$$
\begin{aligned}
& b^{\prime}=b / l, b_{y}^{\prime}=b_{y} / l, c^{\prime}=c /\left(m p_{0} l^{2}\right), h^{\prime}=h / l, k^{\prime}=k /\left(m p_{0}^{2} l^{2}\right), V_{0}^{\prime}=V_{0} /\left(p_{0} l\right), \\
& t^{\prime}=p_{0} t, \quad \rho_{a}^{\prime}=\rho_{a} l^{3} / m, \Omega^{\prime}=\Omega / p_{0}, \omega^{\prime}=\omega / p_{0} \quad\left[p_{0}=\sqrt{g / l}\right]
\end{aligned}
$$

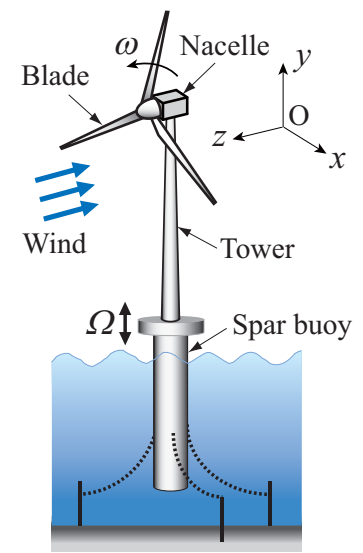

Fig. 1. Spar-type floating offshore wind turbine.

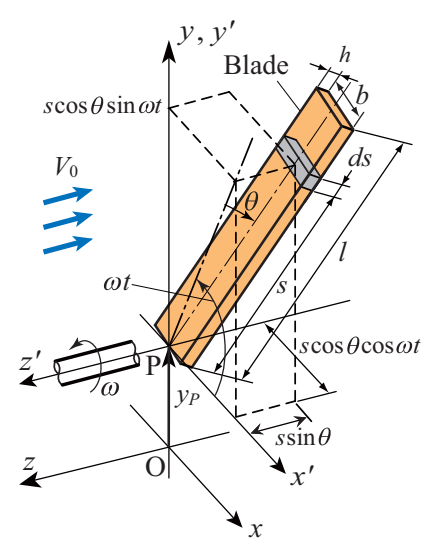

Fig. 2. Theoretical model of a blade. 
are applied to Eq. (1) to obtain the dimensionless equation of motion as

$$
\frac{1}{3} \ddot{\theta}+c \dot{\theta}+k \theta-\frac{1}{2}\left(1-b_{y} \Omega^{2} \sin \Omega t\right) \sin \omega t \sin \theta+\frac{1}{6} \omega^{2} \sin 2 \theta-\frac{1}{2} \rho_{a} b V_{0}^{2} \cos ^{2} \theta=0 .
$$

Note that all primes in Eq. (2) are omitted in Eq. (3) and hereafter for simplicity although the quantities are still dimensionless in the theoretical analysis and results.

\subsection{Unstable regions of linearized system}

When a linear model of the blade is considered, the corresponding linearized system of Eq. (3) is given as

$$
\frac{1}{3} \ddot{\theta}+c \dot{\theta}+\left[k+\frac{1}{3} \omega^{2}-\frac{1}{2}\left(1-b_{y} \Omega^{2} \sin \Omega t\right) \sin \omega t\right] \theta=0 .
$$

The natural frequency $p$ of the system without parametric excitations in Eq. (4) is given as

$$
p=\sqrt{3 k+\omega^{2}} \text {. }
$$

A Mathieu's equation [3] includes a single parametric excitation term. For example, it is well known that unstable vibrations may appear in a pendulum whose supporting point vertically moves [4]. Equation (4), however, includes parametric excitation terms with different three frequencies, i.e., $\omega, \omega+\Omega$, and $\omega-\Omega$. By conducting numerical simulations of Eq. (4), it is found that the following unstable vibrations, each of which includes a predominant frequency, appear as follows:

$$
(\Omega-\omega) / 2,(\Omega+\omega) / 2,(\Omega-2 \omega) / 2,(\Omega+2 \omega) / 2,(\Omega-3 \omega) / 2,(\Omega+3 \omega) / 2, \ldots
$$

These unstable vibrations appear at different rotational speed ranges, hereafter referred to as unstable regions. Because periodic vibrations of predominant frequencies may also appear, the following empirical approach, based on the simulation results, is used to determine the boundaries of the unstable regions. The first-order approximate solution for the periodic vibration of $(\Omega-\omega) / 2$ is assumed as

$$
\theta=a_{1} \cos [(\Omega-\omega) / 2] t+a_{2} \sin [(\Omega-\omega) / 2] t
$$

Substituting Eq. (7) into Eq. (4) generates the frequency components $(\Omega+\omega) / 2,(\Omega+3 \omega) / 2$, $(\Omega-3 \omega) / 2, \quad(3 \Omega+\omega) / 2$, and $(3 \Omega-3 \omega) / 2$. A further substitution of the second-order approximate solution, including these frequency components, into Eq. (4), subsequently generates additional frequency components $(\Omega \pm 5 \omega) / 2,(3 \Omega+3 \omega) / 2,(3 \Omega \pm 5 \omega) / 2,(5 \Omega-\omega) / 2$, $(5 \Omega+3 \omega) / 2$, and $(5 \Omega-5 \omega) / 2$. A final substitution of the third-order approximate solution, including all the generated frequency components, into Eq. (4), results in the equation

$$
A_{1} x_{1}=0
$$

where $\boldsymbol{x}_{1}$ is a vector whose elements are the amplitudes of the frequency components, and $\boldsymbol{A}_{1}$ is a matrix whose elements consist of the system parameters. The condition for the nontrivial solutions of the periodic vibrations is given as

$$
\operatorname{det}\left(\boldsymbol{A}_{1}\right)=0 \text {. }
$$

Equation (9) can be used to determine the boundaries of the unstable regions. The boundaries for the unstable regions of other periodic vibrations of predominant frequencies such as $(\Omega-2 \omega) / 2$ and $(\Omega-3 \omega) / 2$ can also be determined using the same empirical approach which resulted in Eq. (9).

\section{Numerical results}

\subsection{Linearized system}




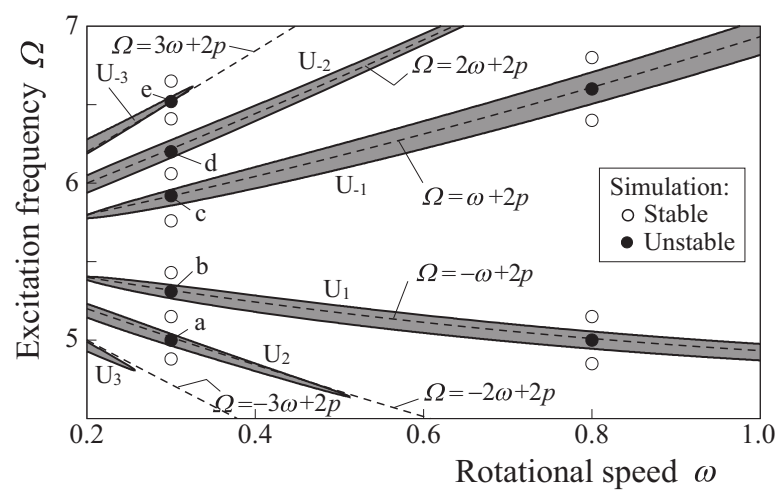

Fig. 3. Unstable regions in the $(\omega, \Omega)$ plane.

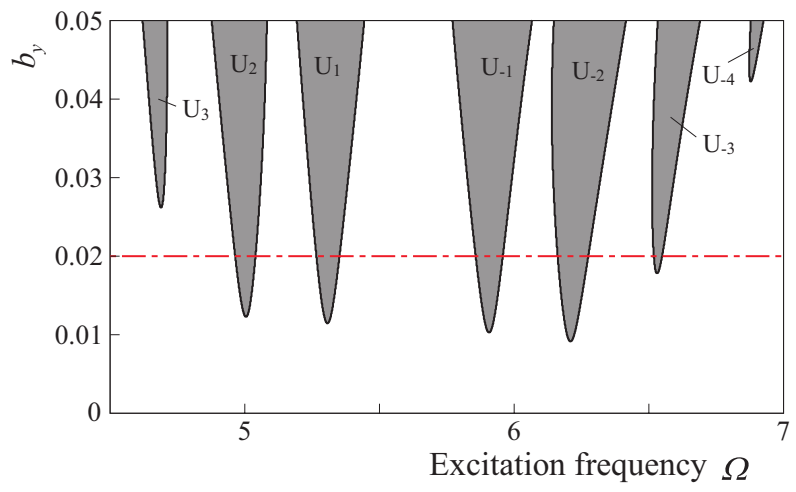

Fig. 5. Unstable regions in the $\left(\Omega, b_{y}\right)$ plane when $\omega=0.3$ in Fig. 3.

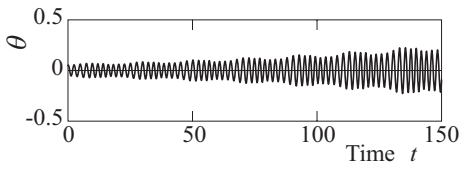

(a) $\Omega=5.00$ in unstable region $\mathrm{U}_{2}$

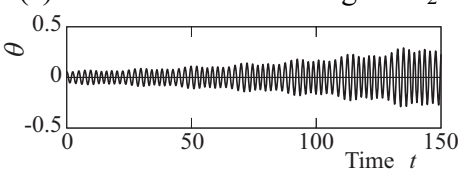

(b) $\Omega=5.31$ in unstable region $\mathrm{U}_{1}$

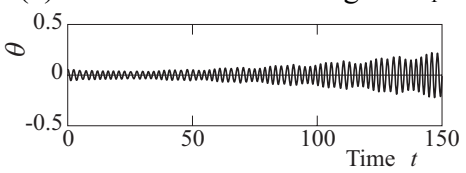

(c) $\Omega=5.92$ in unstable region $\mathrm{U}_{-1}$

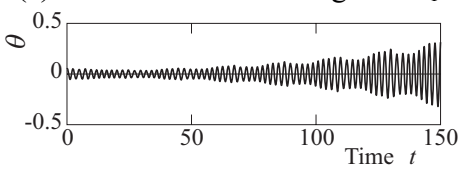

(d) $\Omega=6.20$ in unstable region $\mathrm{U}_{-2}$

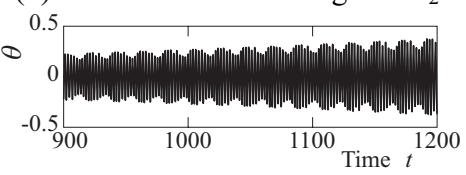

(e) $\Omega=6.52$ in unstable region $\mathrm{U}_{-3}$

Figure 3 shows the numerical results for the linearized system in the $(\omega, \Omega)$ plane when the values of the parameters are $l=1.0, b=0.2, h=0.02, k=2.6, c=0.01$, and $b_{y}=0.02$. Symbols 0 and $\bullet$ represent the stable and unstable vibrations, respectively, calculated from the numerical simulations of Eq. (4). Shaded areas show the unstable regions where unstable vibrations may appear, based on the equations such as Eq. (9). When $p=(\Omega+n \omega) / 2(n= \pm 1$, $\pm 2, \pm 3 \ldots)$ in Eq. (5), theoretical curves can be calculated from the following equation:

$$
\Omega=-n \omega+2 p=-n \omega+2 \sqrt{3 k+\omega^{2}} \quad(n= \pm 1, \pm 2,, \pm 3, \ldots) .
$$

The broken lines represent these curves, and the unstable region corresponding to the curve for $n$ in Eq. (10) is designated as $\mathrm{U}_{n}$. Unstable regions $\mathrm{U}_{1}, \mathrm{U}_{2}$, and $\mathrm{U}_{3}$ appear for $\Omega<5.5$, while unstable regions $U_{-1}, U_{-2}$, and $U_{-3}$ appear for $\Omega>5.5$. Figure 3 demonstrates that the theoretical boundaries of unstable regions are in agreement with the simulation results.

Figure 4 shows the time histories calculated from Eq. (4) at $\omega=0.3$ in Fig. 3. The value of $\Omega$ in Figs. 4(a)-(e) corresponds to the same name in Fig. 3 for unstable regions $\mathrm{U}_{2}, \mathrm{U}_{1}$, $\mathrm{U}_{-1}, \mathrm{U}_{-2}$, and $\mathrm{U}_{-3}$, respectively. The amplitudes of these time histories are irregular and confirm the appearance of unstable vibrations.

Figure 5 shows the unstable regions in the $\left(\Omega, b_{y}\right)$ plane when $\omega=0.3$. The horizontal, dash-dotted line corresponds to Fig. 3 where the wave excitation amplitude $b_{y}$ is 0.02 . The dash-dotted line intersects five unstable regions at nearly the same values of $\Omega$ as in Figs. 3 and 4, corroborating their results. Furthermore, Fig. 5 also effectively demonstrates that the number and width of the unstable regions depend significantly on the value of $b_{y}$, i.e., as the value of $b_{y}$ increases, both the number and width of the unstable regions increase. 


\subsection{Nonlinear system}

Equation (3) includes the nonlinear terms such as $\sin \theta, \sin 2 \theta$ and $\cos ^{2} \theta$. Figure 6 shows the frequency response curves for this nonlinear system. These curves are obtained by applying the swept-sine test to Eq. (3) when $\omega$ and $\omega t$ are replaced by $\lambda t+\omega_{0}$ and $(1 / 2) \lambda t^{2}+\omega_{0} t$, respectively. The vertical axis represents the maximum amplitudes $\theta_{\max }$ of $\theta$ at any instant in time. The values of the parameters are $V_{0}=3.8, \rho_{a}=0.08$, and $\lambda= \pm 1.0 \times 10^{-6}$. Here, $\lambda$ represents the acceleration of the sine sweep excitation. The values of the other parameters are the same as those in Fig. 3. The solid (red) and broken (black) lines represent $\theta_{\max }$ for deceleration and acceleration, respectively. The shaded areas represent the unstable regions obtained from the linear analysis. In Fig. 6(a), when $\Omega=5.0$, the response curves bend to the left and exhibit soft-type nonlinear characteristics, whereas they bend to the right and exhibit hard-type nonlinear characteristics in Fig. 6(b) when $\Omega=6.5$. In both Figs. 6(a) and $6(\mathrm{~b})$, parametric resonances occur near the rotational speeds of the unstable regions.

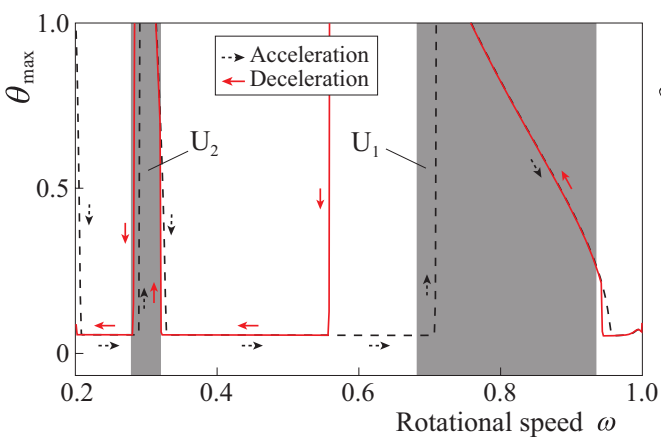

(a) $\Omega=5.0$

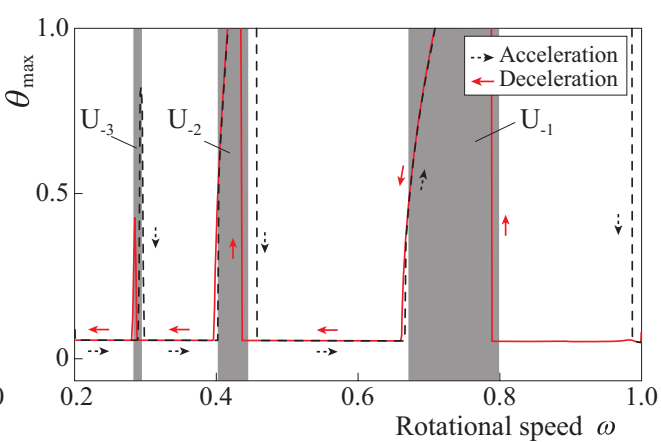

(b) $\Omega=6.5$

Fig. 6. Frequency response curves for Eq. (3).

\section{Experimental results}

In order to confirm the validity of the theoretical results, experiments were conducted. Figure 7 shows a sketch of the experimental setup. A blade consisting of a solid body and spring steel was fixed to a horizontal, rotating shaft which contained pulley 1 . A motor was used to power a flat belt between pulleys 1 and 2. Pulley 1 was mounted on a parallelogrammatic link, and an exciter was used to simulate vertical wave motion. The acceleration of the blade was measured by a wireless motion sensor attached to the blade. The dimensions of the experimental apparatus are listed in Table 1.

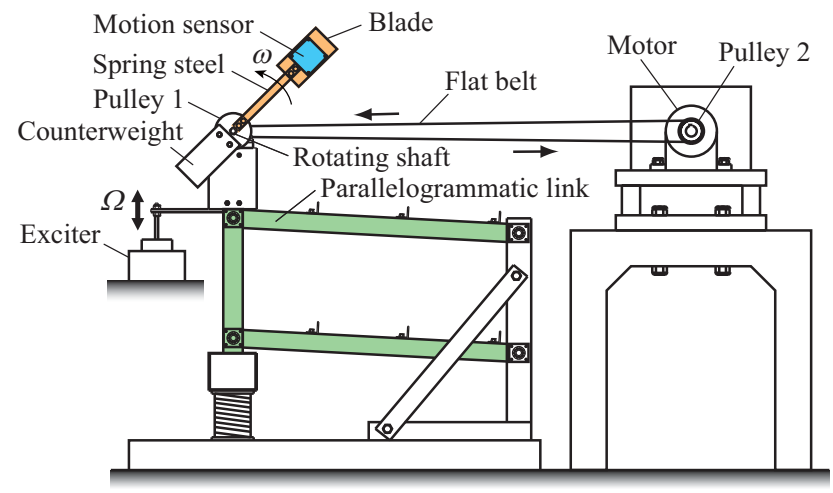

Table 1. Dimensions of the experimental apparatus.

Blade:

Mass $m=0.1687 \mathrm{~kg}$

Length $l=0.09 \mathrm{~m}$

Width $b=0.05 \mathrm{~m}$

Thickness $h=0.005 \mathrm{~m}$

Spring steel:

Spring stiffness $k=6.01 \mathrm{Nm} / \mathrm{rad}$

Damping coefficient $c=8.89 \times 10^{-6} \mathrm{Nms} / \mathrm{rad}$

Motion sensor:

Mass $0.0456 \mathrm{~kg}$

Fig. 7. Sketch of the experimental setup. 


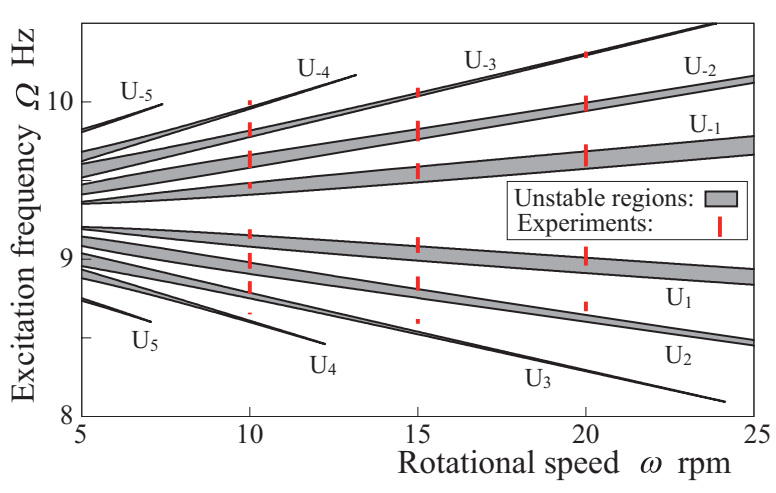

Fig. 8. Parametric resonances observed in the experiments and the corresponding unstable regions.

Figure 8 shows a comparison between the theoretical and experimental results in the $(\omega, \Omega)$ plane when the wave excitation amplitude was $b_{y}=1.6 \mathrm{~mm}$. The shaded areas show the unstable regions where unstable vibrations appeared in the theoretical analysis of the linearized system. In experiments, parametric resonances were observed at $\omega=10,15$, and $20 \mathrm{rpm}$ for several values of the wave excitation frequency $\Omega$, as shown by the vertical (red) lines. Because the experimental data for parametric resonances were observed near the unstable regions, it can be concluded that the theoretical analysis for a linearized system can be used to predict where parametric resonances may occur in a nonlinear system.

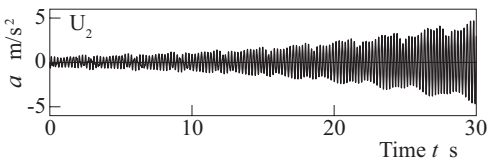

(a) $\Omega=8.67 \mathrm{~Hz}$ in unstable region $\mathrm{U}_{2}$

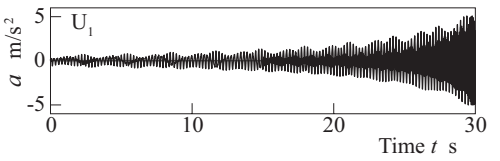

(b) $\Omega=8.96 \mathrm{~Hz}$ in unstable region $\mathrm{U}_{1}$

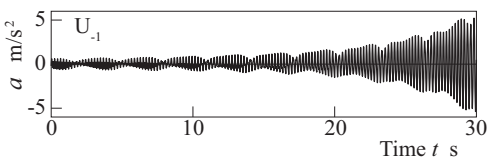

(c) $\Omega=9.59 \mathrm{~Hz}$ in unstable region $\mathrm{U}_{-1}$

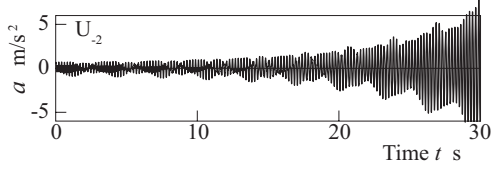

(d) $\Omega=9.94 \mathrm{~Hz}$ in unstable region $\mathrm{U}_{-2}$

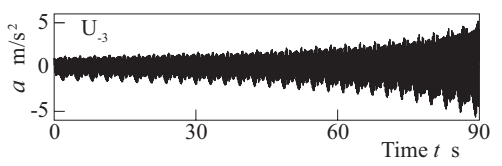

(e) $\Omega=10.28 \mathrm{~Hz}$ in unstable region $\mathrm{U}_{-3}$

Fig. 9. Time histories of parametric resonances at $\omega=20 \mathrm{rpm}$ in Fig 8.

Figures 9(a)-(e) show the time histories observed at $\omega=20 \mathrm{rpm}$ in Fig. 8, when the wave excitation frequencies were $\Omega=8.67,8.96,9.59,9.94$, and $10.28 \mathrm{~Hz}$, respectively. These time histories confirm the appearance of parametric resonances.

\section{Conclusion}

The parametric resonances of a floating wind turbine blade were theoretically and experimentally investigated. In the corresponding linearized system, unstable vibrations appeared at several rotational speeds $\omega$ when the natural frequency $p$ of the system satisfied the condition $p=(\Omega+n \omega) / 2 \quad(n= \pm 1, \pm 2, \pm 3, \ldots)$. In the nonlinear system, parametric resonances occurred near the rotational speeds and wave excitation frequencies of the unstable regions predicted by the theoretical analysis of the corresponding linear system. Experiments further confirmed the validity of the theoretical analysis and results.

\section{References}

[1] B. Battaglia, F. Gorintin, H. Mouslim, Floating offshore wind market outlook, Bilbao Marine Energy Week (2015)

[2] H.P. Lee, Computers \& Structures, 53(2), 351-355 (1994)

[3] L. Meirovitch, Elements of vibration analysis, $2^{\text {nd }}$ Edition (McGraw-Hill, NY, 1986)

[4] M.J. Clifford, S.R. Bishop, J. Austral. Math. Soc., Ser. B, 37(3), 309-319 (1996) 